VOLUME 26 (2019) 1-12

DOI: $10.24330 /$ ieja. 586838

\title{
SOME REMARKS ON THE ORDER SUPERGRAPH OF THE POWER GRAPH OF A FINITE GROUP
}

\author{
A. Hamzeh and A. R. Ashrafi \\ Received: 4 May 2018; Accepted: 26 April 2019 \\ Communicated by Abdullah Harmancı
}

\begin{abstract}
Let $G$ be a finite group. The main supergraph $\mathcal{S}(G)$ is a graph with vertex set $G$ in which two vertices $x$ and $y$ are adjacent if and only if $o(x) \mid o(y)$ or $o(y) \mid o(x)$. In an earlier paper, the main properties of this graph was obtained. The aim of this paper is to investigate the Hamiltonianity, Eulerianness and 2-connectedness of this graph.
\end{abstract}

Mathematics Subject Classification (2010): 05C25, 05C50

Keywords: Power graph, main supergraph, 2-connectivity, Hamiltonian

\section{Introduction}

Let $G$ be a finite group and $x \in G$. The order of $x$ is denoted by $o(x)$ and the least common multiple of all element orders in $G$ is the exponent of $G$ which is denoted by $\operatorname{Exp}(G)$. If there is an element $a \in G$ such that $o(a)=\operatorname{Exp}(G)$, then $G$ is called full exponent. The set of all element orders of $G$ is denoted by $\pi_{e}(G)$ and the set of all prime factors of $|G|$ is denoted by $\pi(G)$. Set $\Xi_{i}(G)$ to be the set of all elements of $G$ of order $i$ and $\Omega_{i}(G)=\left|\Xi_{i}(G)\right|$. Also $n s e(G)=\left\{\Omega_{i}(G) \mid i \in \pi_{e}(G)\right\}$. An EPPO-group is a group that all elements have prime power order and an EPOgroup is a group with elements of prime order.

Throughout this paper graph means simple graph. Suppose $\Gamma$ is such a graph. The number of vertices adjacent to $x$ is the degree of $x$ and is denoted by $\operatorname{deg}_{\Gamma}(x)$. If the graph $\Gamma$ can not be disconnected by removing less than $k$ vertices, then $\Gamma$ is called $k$-connected. It is clear that every Hamiltonian graph is 2 -connected. A set of all vertices in $\Gamma$ such that no two of which are adjacent is an independent set for $\Gamma$. The independent number of $\Gamma, \alpha(\Gamma)$, is the cardinality of an independent set with maximum size. A set $S$ of vertices of a graph $\Gamma$ is a vertex cover for $\Gamma$, if every edge of $\Gamma$ has at least one vertex in $S$ as an endpoint. The vertex cover

The second author was partially supported by the University of Kashan under grant number no $364988 / 444$. 
number, $\beta(\Gamma)$, is the size of a minimum vertex cover of graph. In the graph $\Gamma$ with $n$ vertices always we have $\alpha(\Gamma)+\beta(\Gamma)=n$.

The directed power graph of a group $G$ is a graph with vertex set $G$ and there is a directed edge connecting $x$ to $y$ if and only if $y$ is a power of $x$. This directed graph was introduced in the seminal paper of Kelarev and Quinn in 1999 [13]. In the mentioned paper, the authors considered the directed power graph of groups and gave a complete description of the structure of this graph for a finite abelian group. The same authors [15], extended their results to all semigroups. We refer to $[14,16]$, for some properties of the directed power graph of semigroups.

Suppose $A$ is a simple graph and $\mathcal{G}=\left\{\Gamma_{a}\right\}_{a \in A}$ is a set of graphs labeled by vertices of $A$. Following Sabidussi [20, p. 396], the $A$-join of $\mathcal{G}$ is the graph $\Delta$ with the following vertex and edge sets:

$$
\begin{aligned}
& V(\Delta)=\left\{(x, y) \mid x \in V(A) \& y \in V\left(\Gamma_{x}\right)\right\}, \\
& E(\Delta)=\left\{(x, y)\left(x^{\prime}, y^{\prime}\right) \mid x x^{\prime} \in E(A) \text { or else } x=x^{\prime} \& y y^{\prime} \in E\left(\Gamma_{x}\right)\right\} .
\end{aligned}
$$

If $A$ is an $p$-vertex labeled graph then the $A$-join of $\Delta_{1}, \Delta_{2}, \ldots, \Delta_{p}$ is denoted by $A\left[\Delta_{1}, \Delta_{2}, \ldots, \Delta_{p}\right]$.

The undirected power graph of a finite group $G, \mathcal{P}(G)$, was introduced by Chakrabarty et al. [4]. This graph has $G$ as its vertex set and two vertices $x$ and $y$ are adjacent if and only if one is a power of the other. The main properties of this graph were investigated by Cameron [2] and Cameron and Ghosh [3]. Define the graph $\mathcal{S}(G)$ with vertex set $G$ such that two vertices $x$ and $y$ are adjacent if and only if $o(x) \mid o(y)$ or $o(y) \mid o(x)$. This graph is called the main supergraph of $\mathcal{P}(G)$. Some basic properties of this graph are studied in [11]. In [9], the automorphism group of this graph computed in general and in [10] its eigenvalues and Laplacian eigenvalues were computed. Set $\pi_{e}(G)=\left\{a_{1}, \ldots, a_{k}\right\}$ and define the graph $\Delta_{G}$ with vertex set $\pi_{e}(G)$ and edge set $E\left(\Delta_{G}\right)=\left\{x y\left|x, y \in \pi_{e}(G), x\right| y\right.$ or $\left.y \mid x\right\}$. In $[8,9]$, the authors proved that $\mathcal{S}(G)=\Delta_{G}\left[K_{\Omega_{a_{1}}(G)}, \ldots, K_{\Omega_{a_{k}}(G)}\right]$, where $K_{n}$ denotes the complete graph on $n$ vertices.

The proper power graph $\mathcal{P}^{*}(G)$ and its proper main supergraph $\mathcal{S}^{*}(G)$ are defined as graphs constructed from $\mathcal{P}(G)$ and $\mathcal{S}(G)$ by removing identity element of $G$, respectively.

Suppose $G$ is a finite group, $X \subseteq G$ and $C \subseteq G-\{1\}$. Following Williams [25], the prime graph $\Lambda(G)$ is a simple graph that vertices are primes dividing the order of the group. Two vertices $p$ and $q$ are adjacent if and only if $G$ contains an element of order $p q$. The commuting graph $C(G, X)$ is a simple graph with vertex set $X$, 
and two vertices $x, y \in X$ are adjacent, whenever $x y=y x$. In this paper, we will assume that $X=G-\{1\}$ and the corresponding commuting graph is denoted by $\Delta(G)$. The directed Cayley graph $\overrightarrow{X(G, C)}$ is a graph with vertex set $G$ and edge set $\left\{(g, h) \mid g^{-1} h \in C \cup C^{-1}\right\}$. It is well-known that Cayley graphs are regular and vertex-transitive.

Suppose $\Gamma_{1}$ and $\Gamma_{2}$ are two graphs. The Cartesian product $\Gamma_{1}$ and $\Gamma_{2}, \Gamma_{1} \square \Gamma_{2}$, is a graph with vertex set $V\left(\Gamma_{1}\right) \times V\left(\Gamma_{2}\right)$ such that two vertices $(a, b)$ and $(x, y)$ are adjacent in $\Gamma_{1} \square \Gamma_{2}$ if $a=x$ and $b y \in E(H)$ or $b=y$ and $a x \in E(G)$. The tensor product $\Gamma_{1} \times \Gamma_{2}$ of graphs $\Gamma_{1}$ and $\Gamma_{2}$ is a graph with the same vertex set $V\left(\Gamma_{1}\right) \times V\left(\Gamma_{2}\right)$ and two vertices $(a, b)$ and $(x, y)$ are adjacent in $\Gamma_{1} \times \Gamma_{2}$ if and only if $b y \in E(H)$ and $a x \in E(G)$.

Let $\Gamma$ be a graph and $M \subseteq V(\Gamma) . M$ is called a module if for any $x \notin M$, $M \subseteq N(x)$ or $M \cap N(x)=\emptyset$. The trivial modules are empty set, singletons and the whole set $V$. A graph in which all modules are trivial is said to be primitive. A strong module is a module $M$ such that for any other module $M^{\prime}$, either $M \cap M^{\prime}=\emptyset$ or $M \subseteq M^{\prime}$ or $M^{\prime} \subseteq M$. We now assume that $M$ and $M^{\prime}$ two disjoint modules. If any vertex of $M$ is adjacent to all vertices of $M^{\prime}$, then we say $M$ and $M^{\prime}$ are adjacent, and if there is no an edge such that one of its end points is belong to $M$ and another in $M^{\prime}$ then we say $M$ and $M^{\prime}$ are non-adjacent.

For a module $M$, if $M \subset S$ and there is no module $M^{\prime}$ such that $M \subset M^{\prime} \subset S$, then the module $M$ is maximal with respect to a set $S$ of vertices. We shall assume $S=V$, if the set $S$ is not specified. Let for $1 \leq i \leq k, M_{i}$ be a module of graph $\Gamma$ and $P=\left\{M_{1}, \ldots, M_{k}\right\}$ be a partition of the vertex set of a graph, then $P$ is a modular partition of $\Gamma$. A non-trivial modular partition $P=\left\{M_{1}, \ldots, M_{k}\right\}$ which only contains maximal strong modules is a maximal modular partition. Notice that each graph has a unique maximal modular partition. Quotient graph whose vertices are modules belonging to the modular partition $P$ of graph $\Gamma$ is denoted by $\Gamma / P$. In this graph, two vertices of $\Gamma / P$ are adjacent if and only if the corresponding modules are adjacent in $\Gamma[7]$.

Theorem 1.1. (Modular Decomposition Theorem) [5,6] For any graph $\Gamma$, one of the following three conditions is satisfied:

- $\Gamma$ is not connected.

- $\bar{\Gamma}$ is not connected.

- $\Gamma$ and $\bar{\Gamma}$ are connected and the quotient graph $\Gamma / P$, with $P$ the maximal modular partition of $\Gamma$, is a primitive graph. 
Throughout this paper we refer to [19] for group theory concepts and for graph theoretical concepts and notations, we refer to [24]. For the sake of completeness, in what follows we mention the presentation of the dihedral group $D_{2 n}$, the semidihedral group $S D_{8 n}$, the dicyclic group $T_{4 n}$ and the group $V_{8 n}$.

$$
\begin{aligned}
D_{2 n} & =\left\langle a, b \mid a^{n}=b^{2}=e, b a b=a^{-1}\right\rangle, \\
S D_{8 n} & =\left\langle a, b \mid a^{4 n}=b^{2}=e, b a b=a^{2 n-1}\right\rangle, \\
T_{4 n} & =\left\langle a, b \mid a^{2 n}=1, a^{n}=b^{2}, b^{-1} a b=a^{-1}\right\rangle, \\
V_{8 n} & =\left\langle a, b \mid a^{2 n}=b^{4}=e, a b a=b^{-1}, a b^{-1} a=b\right\rangle .
\end{aligned}
$$

It is easy to see the dicyclic group $T_{4 n}$ has order $4 n$ and the groups $S D_{8 n}$ and $V_{8 n}$ have order $8 n$.

\section{Main results}

A vertex in a graph $\Gamma$ is said to be even, if its degree is an even integer. There is a condition for Eulerian of a graph $\Gamma$ which states that $\Gamma$ is Eulerian if and only if all of its degrees are even.

Theorem 2.1. Let $G$ be a finite group. The graph $\mathcal{S}(G)$ is Eulerian if and only if $G$ is an odd order group.

Proof. Suppose $G$ is a group of order $n$. Then the degree of identity has to be $n-1$ and so $n$ is odd. Conversely, suppose $n$ is odd and $\pi_{e}(G)=\left\{a_{1}, \ldots, a_{k}\right\}$. Choose the non-identity vertex $x$ in $\mathcal{S}(G)$ and assume that $o(x)=a_{i}$. Then

$$
\operatorname{deg}_{\mathcal{S}(G)}(x)=\Omega_{a_{i}}(G)+\sum_{a_{i} \mid a_{j} \neq \text { or }} \sum_{\left(a_{j} \mid a_{i} \& a_{i} \neq a_{j}\right)} \Omega_{a_{j}}(G) .
$$

If $k_{i}, 1 \leq i \leq k$, denotes the number of cyclic subgroups of order $a_{i}$ then $\Omega_{a_{i}}(G)=$ $k \phi\left(a_{i}\right)$, that $\phi$ is the Euler's totient function. Since $G$ has odd order, it does not have involutions and $\phi(m), m \geq 3$, is even. Thus for each $a_{i}, a_{i} \in \pi_{e}(G), \Omega_{a_{i}}(G)$ is even. Therefore, degree of every vertex in $\mathcal{S}(G)$ is even and $\mathcal{S}(G)$ is Eulerian.

In the next theorem, the relationship between connectedness of $\mathcal{S}^{*}(G)$ and $\Lambda(G)$ is studied.

Theorem 2.2. ([11]) If the prime graph of a group $G$ is disconnected then $\mathcal{S}^{*}(G)$ is disconnected. In particular, $\mathcal{S}(G)$ is not Hamiltonian.

Theorem 2.3. Let $G$ be a finite group. If $\Delta_{G}$ is Hamiltonian then $\mathcal{S}(G)$ will be Hamiltonian. 
Proof. Suppose $\Delta_{G}$ is Hamiltonian and $T: e \sim a_{1} \sim \ldots \sim a_{k} \sim e$ is a Hamiltonian cycle in $\Delta_{G}$. Set $\Xi(G)=\left\{x_{i 1}, x_{i 2}, \ldots, x_{i \Omega_{a_{i}}(G)}\right\}$. We construct a Hamiltonian cycle $T^{\prime}$ in $\mathcal{S}(G)$ as follows:

$$
\begin{aligned}
T^{\prime}: & e \sim x_{11} \sim \ldots \sim x_{1 \Omega_{a_{1}}(G)} \sim x_{21} \sim \ldots \sim x_{2 \Omega_{a_{2}}(G)} \sim \ldots \sim \\
& x_{k 1} \sim \ldots \sim x_{k \Omega_{a_{k}}(G)} \sim e,
\end{aligned}
$$

and so $\mathcal{S}(G)$ is Hamiltonian, as desired.

Corollary 2.4. The main supergraph of the power graph of the following simple groups are not Hamiltonian:

(1) ${ }^{2} F_{4}(q)$, where $q=2^{2 m+1}$ and $m \geq 1$;

(2) ${ }^{2} G_{2}(q)$, where $q=3^{2 m+1}$ and $m \geq 0$;

(3) $A_{1}(q), A_{2}(q), B_{2}(q), C_{2}(q)$ and $S_{4}(q)$, where $q$ is an odd prime power;

(4) $F_{4}\left(2^{m}\right), m \geq 1$ and $U_{3}(q)$, where $q$ is a prime power.

Proof. Apply Theorems 2.34 and 2.35 from [11].

It is easy to see that the main supergraph of the power graph of the cyclic group of order $p, p$ is prime, is Hamiltonian. This simple result and Corollary 2.4 suggest the following conjecture:

Conjecture 2.5. The main supergraph of the power graph of a non-abelian finite simple group is not Hamiltonian.

Theorem 2.6. If $G$ is full exponent then $\mathcal{S}(G)$ is 2-connected.

Proof. Suppose $x$ is an element of order $\operatorname{Exp}(G)$. Then $e$ and $x$ are adjacent to all elements of the group. This proves that $\mathcal{S}(G)$ is 2-connected.

Theorem 2.7. If $G$ is an abelian group, then $\mathcal{S}(G)$ is 2-connected.

Proof. To prove the theorem, it is enough to show that $\mathcal{S}^{*}(G)$ is connected. Choose non-identity elements $x, y \in \mathcal{S}^{*}(G)$. Since $G$ is abelian, $x y=y x$. If $x$ and $y$ are adjacent in $\mathcal{S}(G)$, then are adjacent in $\mathcal{S}^{*}(G)$ too. This implies that $o(x) \nmid o(y)$ and $o(y) \nmid o(x)$. Our main proof will consider the following two cases:

(1) $o(x)$ and $o(y)$ are coprime. Since $o(x y)=o(x) o(y), o(x) \mid o(x y)$ and $o(y) \mid$ $o(x y)$. Thus $x \sim x y \sim y$ is a path in $\mathcal{S}^{*}(G)$ and so $x, y$ are vertices of a connected component of $\mathcal{S}^{*}(G)$.

(2) $n=(o(x), o(y)) \neq 1$. Without loss of generality, we can assume that $o(x)>o(y)$. Since $o(x) \equiv n(\bmod o(y)), y^{n}=(x y)^{o(x)}$. On the other hand, 
$x^{o(y)}=(x y)^{o(y)}$ and so $y \sim y^{n} \sim(x y)^{o(x)} \sim x y \sim(x y)^{o(y)} \sim x^{o(y)} \sim x$ is a path in $\mathcal{S}^{*}(G)$. Hence $x$ and $y$ are in a connected component of $\mathcal{S}^{*}(G)$.

This proves that $\mathcal{S}^{*}(G)$ is connected.

Lemma 2.8. Let $G$ and $H$ be groups such that $(|G|,|H|)=1$. Then $\mathcal{S}(G \times H)=$ $\mathcal{S}(G) \times \mathcal{S}(H)$.

Proof. Suppose $(x, y)$ and $(a, b)$ are adjacent vertices in $\mathcal{S}(G \times H)$. Then $o((x, y)) \mid$ $o((a, b))$ or $o((a, b)) \mid o((x, y))$. Since $G$ and $H$ have coprime order, $o(x) o(y) \mid$ $o(a) o(b)$ or $o(a) o(b) \mid o(x) o(y)$. On the other hand, $(o(a), o(y))=(o(b), o(x))=$ 1. Hence $o(a) o(b) \mid o(x) o(y)$ implies that $o(a) \mid o(x)$ and $o(b) \mid o(y)$. Similarly, $o(x) o(y) \mid o(a) o(b)$ implies that $o(x) \mid o(a)$ and $o(y) \mid o(b)$. Therefor, $a, x$ are adjacent in $\mathcal{S}(G)$, and $b, y$ are adjacent in $\mathcal{S}(H)$ which proves that $(a, b)$ and $(x, y)$ are adjacent in $\mathcal{S}(G) \times \mathcal{S}(H)$. A similar argument as above shows that if $(a, b)$ and $(x, y)$ are adjacent in $\mathcal{S}(G) \times \mathcal{S}(H)$, then $a x \in E(\mathcal{S}(G))$ and by $\in E(\mathcal{S}(H))$.

The proof of the previous lemma shows that in general $\mathcal{S}(G) \times \mathcal{S}(H)$ is a subgraph of $\mathcal{S}(G \times H)$. By [12, Theorem 5.29], if $G$ and $H$ are non-empty graphs, then $G \times H$ is connected if and only if both of $G$ and $H$ are connected and at least one of them are non-bipartite. Moreover, if $G$ and $H$ are connected and bipartite, then $G \times H$ has exactly two connected components. In the following theorem, we apply this result to prove that the main supergraph of the power graph of a nilpotent group is 2-connected.

Theorem 2.9. If $G$ is nilpotent, then $\mathcal{S}(G)$ is 2-connected.

Proof. Since $G$ is nilpotent, $G \cong P_{1} \times \ldots \times P_{r}$, where $P_{i}$ 's are all Sylow $P_{i^{-}}$ subgroups of $G$. By Lemma $2.8, \mathcal{S}(G) \cong \mathcal{S}\left(P_{1} \times \ldots \times P_{r}\right)=\mathcal{S}\left(P_{1}\right) \times \ldots \times \mathcal{S}\left(P_{r}\right)$ and so $\mathcal{S}^{*}(G) \cong \mathcal{S}^{*}\left(P_{1} \times \ldots \times P_{r}\right)=\mathcal{S}^{*}\left(P_{1}\right) \times \ldots \times \mathcal{S}^{*}\left(P_{r}\right)$. Since $\mathcal{S}^{*}\left(P_{i}\right), 1 \leq i \leq r$, are complete, they are non-bipartite and connected. This shows that $\mathcal{S}^{*}(G)$ is connected, as desired.

Theorem 2.10. Let $G$ be a finite group. If $x y \in E(\Delta(G))$ then $x$ and $y$ are in the same component of $\mathcal{S}^{*}(G)$.

Proof. By definition, $V(\Delta(G))=V\left(\mathcal{S}^{*}(G)\right)$. Suppose, $x, y$ are adjacent vertices of $\Delta(G)$. So $x y=y x$. If $o(x) \mid o(y)$ or $o(y) \mid o(x)$ then $x$ and $y$ are adjacent in $\mathcal{S}^{*}(G)$. We now assume that $o(x) \nmid o(y)$ and $o(y) \nmid o(x)$. We consider two cases that $(o(x), o(y))=1$ or $(o(x), o(y)) \neq 1$.

(1) $(o(x), o(y))=1$. In this case, $o(x y)=o(x) o(y)$. This gives a path $x \sim$ $x y \sim y$ in $\mathcal{S}^{*}(G)$, as desired. 
(2) $(o(x), o(y)) \neq 1$. Choose the prime number $p$ such that $p \mid o(x)$ and $p \mid o(y)$. If $t \in G$ has order $p$ then $x \sim t \sim y$ is a path in $\mathcal{S}^{*}(G)$.

This completes the proof.

Corollary 2.11. If $\Delta(G)$ is complete then $\mathcal{S}(G)$ is 2-connected.

It is clear that if $G$ and $H$ are groups with the same order such that for each divisor $d$ of $|G|, \Omega_{d}(G)=\Omega_{d}(H)$ then $\mathcal{S}(G) \cong \mathcal{S}(H)$. The converse of this result is not generally correct. To prove, we consider $G=Z_{4} \times Z_{4}$ and $H=Z_{2} \times Z_{4} \times Z_{2}$. Since $G$ and $H$ are 2-groups, $\mathcal{S}(G) \cong \mathcal{S}(H)$. But $\Omega_{4}(G)=8<12=\Omega_{4}(H)$ and $\Omega_{2}(G)=7>3=\Omega_{2}(H)$. On the other hand, it is possible to find finite groups $G$ and $H$ such that $\mathcal{S}(G) \cong \mathcal{S}(H)$, but $\pi_{e}(G) \neq \pi_{e}(H)$. An example is the pair $(G, H)=\left(D_{8}, Z_{8}\right)$. Finally, it is possible to construct the pair $(G, H)$ of finite groups such that $\pi_{e}(G)=\pi_{e}(H)$, but $\mathcal{S}(G) \nsucceq \mathcal{S}(H)$. To see this, it is enough to assume that $G=D_{20}$ and $H=Z_{2} \times Z_{10}$. In what follows, we prove that in the group under same specific conditions the equality of spectrum and order implies that the main supergraph are isomorphic.

Theorem 2.12. (See $[1,23]$ ). Suppose $G_{1}$ is a finite group and $G_{2}$ is one of the following finite groups:

(1) A finite simple group,

(2) A symmetric group $S_{n}, n \geq 3$,

(3) Automorphism group of a sporadic simple group,

then $G_{1} \cong G_{2}$ if and only if $\left|G_{1}\right|=\left|G_{2}\right|$ and $\pi_{e}\left(G_{1}\right)=\pi_{e}\left(G_{2}\right)$.

Corollary 2.13. If $G_{1}$ is a finite group and $G_{2}$ is one of the following finite groups:

(1) A finite simple group,

(2) A symmetric group $S_{n}, n \geq 3$,

(3) Automorphism group of a sporadic simple group.

If $\left|G_{1}\right|=\left|G_{2}\right|$ and $\pi_{e}\left(G_{1}\right)=\pi_{e}\left(G_{2}\right)$ then $\mathcal{S}\left(G_{1}\right) \cong \mathcal{S}\left(G_{2}\right)$.

In the following result, the finite groups $G$ in which the main supergraph $\mathcal{S}(G)$ is vertex transitive are classified.

Theorem 2.14. Let $G$ be a finite group, then $\mathcal{S}(G)$ is a vertex transitive if and only if $G$ is a p-group. There is no group $G$ such that $\overrightarrow{\mathcal{S}(G)}$ is vertex transitive.

Proof. If $G$ is a $p$-group then $\mathcal{S}(G)$ is complete and so it is a Cayley graph. Conversely, we assume that $\mathcal{S}(G)$ is vertex-transitive, where $G$ has order $n$. Since $\operatorname{deg}_{\mathcal{S}(G)}(e)=n-1, \mathcal{S}(G)$ has to be complete and so $G$ is a $p$-group. 
We now assume that $G$ is a finite group such that $\overrightarrow{\mathcal{S}(G)}$ is vertex transitive. Then each vertex of $\overrightarrow{\mathcal{S}(G)}$ will have the in-degree $n-1$ and out-degree zero which is impossible.

The present authors [11], proved that for each finite group $G$ we have $|\pi(G)| \leq$ $\alpha(\mathcal{S}(G)) \leq\left|\pi_{e}(G)\right|-1$ with right-hand equality if and only if $G$ is an EPO-group. Applying this result, we have:

Theorem 2.15. If $G$ is a finite group of order $n$ then $n+1-\left|\pi_{e}(G)\right| \leq \beta(\mathcal{S}(G)) \leq$ $n-|\pi(G)|$. The left-hand equality is attained if and only if $G$ is an EPO-group.

Theorem 2.16. Let $G$ be a finite group. $\overline{\mathcal{S}^{*}(G)}$ is complete if and only if $G \cong Z_{2}$.

Proof. Suppose $\overline{\mathcal{S}^{*}(G)}$ is a complete graph. Then $G$ is an EPO-group and there is a unique elements of each order. So, $\mathcal{S}(G)$ is a star graph and by [11, Corollary $2.18], G \cong Z_{2}$. The converse is obvious.

Theorem 2.17. Let $G$ be a finite group of order $>2$. Then $G$ has full exponent if and only if $\overline{\mathcal{S}^{*}(G)}$ is disconnected.

Proof. If $G$ is full exponent group of order $n, n>2$ [11, Theorem 2.15 ], there are at least two elements of degree $n-1$ in $\mathcal{S}(G)$. This proves that $\overline{\mathcal{S}^{*}(G)}$ is disconnected. To prove the converse, we show that if $G$ is not a full exponent group of order $n, n>2$, then $\overline{\mathcal{S}^{*}(G)}$ is connected. Suppose $|G|=p_{1}{ }^{n_{1}} \ldots p_{k}{ }^{n_{k}}$. If $k=1$, then $\mathcal{S}(G)$ is complete and $\overline{\mathcal{S}^{*}(G)}$ is an empty graph, as desired. Suppose $k \geq 2$. Define

$$
V_{i}=\left\{g \in G|1 \neq o(g)| p_{i}^{n_{i}}\right\} .
$$

Then for each $i$, the graph $\overline{\mathcal{S}^{*}(G)}$ has an induced subgraph isomorphic to $\overline{K_{\left|V_{i}\right|}}$ in such a way that every element $x \in V_{i}$ is adjacent to every element $y \in V_{j}$, $i \neq j$. Thus the induced subgraph $\left[\bigcup_{i=1}^{k} V_{i}\right]$ is connected. Suppose $x, y \notin \bigcup_{i=1}^{k} V_{i}$, $o(x)=q_{1}{ }^{\alpha_{1}} \ldots q_{r}{ }^{\alpha_{r}}$ and $o(y)=q_{1}^{\beta_{1}} \ldots q_{s}{ }^{\beta_{s}}, r \leq s$. If $(o(x), o(y))=1$, then $x y \in E\left(\overline{\mathcal{S}^{*}(G)}\right)$ as desired. We now assume that $d=(o(x), o(y)) \neq 1$. If there exists a prime number $p$ such that $p \nmid d$ then we choose an element $z$ of order $p$ in $G$. So $x \sim z \sim y$ is a path connecting $x$ and $y$ in $\mathcal{S}(G)$. Hence, it is enough to assume that, for any $i, 1 \leq i \leq k, p_{i} \mid d$. Suppose $o(x)=p_{1}^{\gamma_{1}} \ldots p_{k}^{\gamma_{k}}$ and $o(y)=p_{1}{ }^{\delta_{1}} \ldots p_{k}{ }^{\delta_{k}}$. If $o(x) \nmid o(y)$ and $o(y) \nmid o(x)$ then $x y \in E\left(\overline{\mathcal{S}^{*}(G)}\right)$. Suppose $o(x) \mid o(y)$ and choose $i$ such that $\gamma_{i} \neq \alpha_{i}$. Then $x \sim x_{i} \sim y$ is a path in $\overline{\mathcal{S}^{*}(G)}$. This completes the proof.

Theorem 2.18. If $G$ is a full exponent group, then the number of connected components of $\overline{\mathcal{S}^{*}(G)}$ is $c\left(\overline{\mathcal{S}^{*}(G)}\right)=\varphi(G)+1$, where $\varphi(G)=|\{a \in G \mid o(a)=\operatorname{Exp}(G)\}|$. 
Proof. Suppose $G$ is a full exponent group of order $p_{1}{ }^{n_{1}} \ldots p_{k}{ }^{n_{k}}$, where, $p_{i}, 1 \leq$ $i \leq k$ are distinct primes and $k>1$. Similar to the Theorem 2.17, we define

$$
V_{i}=\left\{g \in G|1 \neq o(g)| p_{i}^{n_{i}}\right\} .
$$

By Theorem 2.17, the induced subgraph on $\bigcup_{i=1}^{k} V_{i}$ is connected. Suppose $x, y \notin$ $\bigcup_{i=1}^{k} V_{i}$. If $o(x), o(y) \notin\{|G|, \operatorname{Exp}(G)\}$ then by similar argument as in Theorem 2.17, there exits an element $u \in \bigcup_{i=1}^{k} V_{i}$, such that $x \sim u \sim y$ is path in $\overline{\mathcal{S}^{*}(G)}$. We now assume that $o(x) \in\{|G|, \operatorname{Exp}(G)\}$. Then $\{x\}$ is a component of $\overline{\mathcal{S}^{*}(G)}$ and so the number of connected components is $\varphi(G)+1$.

Theorem 2.19. Let $G$ be a finite group. Then,

(1) if $\operatorname{Exp}(G)=m$, then $c\left(\overline{\mathcal{S}^{*}(G)}\right)=k \phi(m)+1$, where $k$ is the number of cyclic subgroups of order $m$ in $G$;

(2) if $G$ is nilpotent, then $c\left(\overline{\mathcal{S}^{*}(G)}\right)=\prod_{i=1}^{k} \varphi\left(G_{i}\right)+1$, where $G_{i}$ 's are Sylow subgroups of $G$;

(3) $c\left(\overline{\mathcal{S}^{*}(G)}\right)=\phi(|G|)+1$ if and only if the number of cyclic subgroups of order $\operatorname{Exp}(G)$ in $G$ is $\frac{|G|}{\operatorname{Exp}(G)}$.

Proof. Apply Theorems 2.2, 2.6, 2.8 and 3.2 from [22].

Corollary 2.20. The following hold:

(1) if $2^{k} \neq n \geq 3$ is an even positive integer then $c\left(\overline{\mathcal{S}^{*}\left(D_{2 n}\right)}\right)=\phi(n)+1$, and if $n$ is odd then $\overline{\mathcal{S}^{*}\left(D_{2 n}\right)}$ is connected;

(2) if $n \geq 3, \overline{\mathcal{S}^{*}\left(S_{n}\right)}$ is connected and if $n \geq 4$, then $\overline{\mathcal{S}^{*}\left(A_{n}\right)}$ is connected;

(3) if $n=2^{k}$, then $c\left(\overline{\mathcal{S}^{*}\left(S D_{8 n}\right)}\right)=8 n-1$ and if $n \neq 2^{k}$, then $c\left(\overline{\mathcal{S}^{*}\left(S D_{8 n}\right)}\right)=$ $\phi(4 n)+1 ;$

(4) if $n$ is odd, then $\overline{\mathcal{S}^{*}\left(T_{4 n}\right)}$ is connected and if $n=2^{k}$, then $c\left(\overline{\mathcal{S}^{*}\left(T_{4 n}\right)}\right)=$ $4 n-1$. If $n \neq 2^{k}$ and $n$ is an even number, then $c\left(\overline{\mathcal{S}^{*}\left(T_{4 n}\right)}\right)=\phi(2 n)+1$;

(5) if $n$ is odd, then $\overline{\mathcal{S}^{*}\left(V_{8 n}\right)}$ is connected. If $n=2^{k}$, then $c\left(\overline{\mathcal{S}^{*}\left(V_{8 n}\right)}\right)=8 n-1$ and if $n \neq 2^{k}$ and $n$ is an even number, then $c\left(\overline{\mathcal{S}^{*}\left(V_{8 n}\right)}\right)=\phi(2 n)+1$.

By the graph structure of $\mathcal{S}(G)=\Delta_{G}\left[K_{\Omega_{a_{1}}(G)}, \ldots, K_{\Omega_{a_{k}}(G)}\right]$ and definition of module, one can see that every $K_{\Omega_{a_{i}}(G)}, 1 \leq i \leq k$, in $\mathcal{S}(G)$ is a maximal strong module. Also $P=\left\{V\left(K_{\Omega_{a_{1}}(G)}\right), \ldots, V\left(K_{\Omega_{a_{k}}(G)}\right)\right\}$ is a modular partition of $\mathcal{S}(G)$ and quotient graph $\mathcal{S}(G) / P$ is isomorphic to $\Delta_{G}$.

Theorem 2.21. Let $G_{1}$ and $G_{2}$ be two finite groups. We also assume that these groups are not full exponent, they are not p-groups, for some prime number $p$, and the graphs $\Delta_{G_{1}}$ and $\Delta_{G_{2}}$ are primitive. If $\mathcal{S}^{*}\left(G_{1}\right) \cong \mathcal{S}^{*}\left(G_{2}\right)$, then $\left|G_{1}\right|=\left|G_{2}\right|$ and $n s e\left(G_{1}\right)=n s e\left(G_{2}\right)$. 
Proof. By Theorem 1.1, since $\Delta_{G_{1}}$ and $\Delta_{G_{2}}$ are primitive, $\mathcal{S}^{*}\left(G_{1}\right), \mathcal{S}^{*}\left(G_{2}\right), \overline{\mathcal{S}^{*}\left(G_{1}\right)}$ and $\overline{\mathcal{S}^{*}\left(G_{2}\right)}$ are connected. In addition, each graph has a unique maximal modular partition and $\mathcal{S}^{*}\left(G_{1}\right) \cong \mathcal{S}^{*}\left(G_{2}\right)$ implies that $\Delta_{G_{1}} \cong \Delta_{G_{2}}$ and so $\left|G_{1}\right|=\left|G_{2}\right|$. This shows that $n \operatorname{se}\left(G_{1}\right)=n \operatorname{se}\left(G_{2}\right)$, as desired.

Theorem 2.22. $[17,18,21]$ Suppose $G, H$ are finite groups and one of the following are satisfied:

- $H$ is a sporadic simple group;

- $H$ is a Mathieu group;

- $H$ is the symmetric group $S_{r}$, where $r$ is prime number.

If $|G|=|H|$ and $n s e(G)=n s e(H)$, then $G \cong H$.

Theorem 2.23. Suppose $G_{1}$ and $G_{2}$ satisfy the conditions of Theorem 2.21. We also assume that one of the following conditions are satisfied:

- $G_{1}$ is a sporadic simple group;

- $G_{1}$ is a Mathieu group;

- $G_{1}$ is the symmetric group $S_{r}$, where $r$ is prime number.

If $\mathcal{S}^{*}\left(G_{1}\right) \cong \mathcal{S}^{*}\left(G_{2}\right)$, then $G_{1} \cong G_{2}$.

Proof. Apply Theorems 2.21 and 2.22 .

Acknowledgement. The authors would like to thank the referee for the valuable suggestions and comments.

\section{References}

[1] J. X. Bi, A characterization of symmetric groups (Chinese), Acta. Math. Sinica, 33 (1990), 70-77.

[2] P. J. Cameron, The power graph of a finite group, II, J. Group Theory, 13 (2010), 779-783.

[3] P. J. Cameron and S. Ghosh, The power graph of a finite group, Discrete Math., 311 (2011), 1220-1222.

[4] I. Chakrabarty, S. Ghosh and M. K. Sen, Undirected power graphs of semigroups, Semigroup Forum, 78 (2009), 410-426.

[5] M. Chein, M. Habib and M. C. Maurer, Partitive hypergraphs, Discrete Math., 37 (1981), 35-50.

[6] T. Gallai, Transitiv orientierbare graphen (German), Acta Math. Acad. Sci. Hungar., 18 (1967), 25-66. 
[7] M. Habib and C. Paul, A survey of the algorithmic aspects of modular decomposition, Comput. Sci. Rev., 4 (2010), 41-59.

[8] A. Hamzeh, Spectrum and L-spectrum of the cyclic graph, Southeast Asian Bull. Math., 42 (2018), 875-884.

[9] A. Hamzeh and A. R. Ashrafi, Automorphism groups of supergraphs of the power graph of a finite group, European J. Combin., 60 (2017), 82-88.

[10] A. Hamzeh and A. R. Ashrafi, Spectrum and L-spectrum of the power graph and its main supergraph for certain finite groups, Filomat, 31(16) (2017), 53235334.

[11] A. Hamzeh and A. R. Ashrafi, The order supergraph of the power graph of a finite group, Turkish J. Math., 42 (2018), 1978-1989.

[12] W. Imrich and S. Klavžar, Product Graphs: Structure and Recognition, Wiley-Interscience Series in Discrete Mathematics and Optimization, WileyInterscience, New York, 2000.

[13] A. V. Kelarev and S. J. Quinn, A combinatorial property and power graphs of groups, Contributions to General Algebra, 12 (Vienna, 1999), Heyn, Klagenfurt, (2000), 229-235.

[14] A. V. Kelarev and S. J. Quinn, Directed graphs and combinatorial properties of semigroups, J. Algebra, 251(1) (2002), 16-26.

[15] A. V. Kelarev and S. J. Quinn, A combinatorial property and power graphs of semigroups, Comment. Math. Univ. Carolin., 45(1) (2004), 1-7.

[16] A. V. Kelarev, S. J. Quinn and R. Smolíková, Power graphs and semigroups of matrices, Bull. Austral. Math. Soc., 63(2) (2001), 341-344.

[17] A. R. Khalili Asboei, S. S. Salehi Amiri, A. Iranmanesh and A. Tehranian, A characterization of symmetric group $S_{r}$, where $r$ is prime number, Ann. Math. Inform., 40 (2012), 13-23.

[18] A. R. Khalili Asboei, S. S. Salehi Amiri, A. Iranmanesh and A. Tehranian, A characterization of sporadic simple groups by nse and order, J. Algebra Appl., 12 (2013), 1250158 (3 pp).

[19] J. S. Rose, A Course on Group Theory, Cambridge University Prees, Cambridge, New York-Melbourne, 1978.

[20] G. Sabidussi, Graph derivatives, Math. Z., 76 (1961), 385-401.

[21] C. Shao and Q. Jiang, A new characterization of Mathieu groups, Arch. Math. (Brno), 46 (2010), 13-23.

[22] M. Tărnăuceanu, A generalization of the Euler's totient function, Asian-Eur. J. Math., 8(4) (2015), 1550087 (13 pp). 
[23] A. V. Vasil'ev, M. A. Grechkoseeva and V. D. Mazurov, Characterization of finite simple groups by spectrum and order, Algebra Logic, 48 (2009), 385-409.

[24] D. B. West, Introduction to Graph Theory, Second Edition, Prentice Hall, Inc., Upper Saddle River, NJ, 2001.

[25] J. S. Williams, Prime graph components of finite groups, J. Algebra, 69 (1981), 487-513.

\section{A. Hamzeh}

Department of Insurance General

Insurance Research Center

Tehran, I. R. Iran

e-mail: hamze2006@yahoo.com, hamzeh@irc.ac.ir

A. R. Ashrafi (Corresponding Author)

Department of Pure Mathematics

Faculty of Mathematical Sciences

University of Kashan

Kashan 87317-53153, I. R. Iran

e-mail: ashrafi@kashanu.ac.ir 